\title{
Enfermeiro de Bordo na Assistência em Obstetrícia no Transporte Aeromédico: Uma Revisão Narrativa
}

\author{
Elia Machado de Oliveira, ${ }^{1}$ Wellington Fernando da Silva Ferreira, ${ }^{2}$ \\ Denecir de Almeida Dutra ${ }^{3}$
}

\begin{abstract}
RESUMO
Objetiva-se com o presente estudo identificar as principais atribuições do profissional enfermeiro de bordo durante o transporte aeromédico e sua dinâmica assistencial no processo gravídico em remoção de emergência. Trata-se de revisão narrativa, descritiva e exploratória. A busca de periódicos nacionais, que correlacionassem a temática, ocorreu em bases de dados: SciELO, Lilacs, Bireme, BVS, publicados entre os anos de 2013 ao primeiro semestre de 2018. Dessa forma, na última fase de seleção, foi realizada a leitura integral de todos os manuscritos e a amostra final ficou constituída por 16 textos científicos. Os principais resultados evidenciados apontam para eixos da temática analisada: o papel do enfermeiro de bordo no atendimento pré-hospitalar durante o transporte aeromédico, avaliação das condições do paciente em processo gravídico, com adequada assistência pautada por meio de protocolos, além de habilidade e capacitação necessárias para a remoção e intercorrências em voo. Transporte aeromédico, portanto, é uma atividade em desenvolvimento no Brasil, e sua temática deve ser aprofundada para a expansão de conhecimento, contribuindo para a rápida intervenção no processo de remoção de paciente em estado de emergência.
\end{abstract}

Palavras-chave: Transporte aéreo de pacientes. Enfermagem de bordo. Processo gravídico.

$$
\text { NURSING NURSERY AT OBSTETRIC CARE IN AEROMETRIC TRANSPORT: A NARRATIVE REVIEW }
$$

\section{ABSTRACT}

The objective of this study is to identify the main duties of the on-board nurse during air ambulance transport and their assistance dynamics in the pregnancy process in emergency evacuation. This is an exploratory descriptive narrative review. The search for national journals, which correlated the theme, occurred in databases: Scielo, Lilacs, Bireme, VHL, published between the years of 2013 and the first half of 2018, in this way, in the last selection phase, of all manuscripts, and final sample consisting of 16 scientific texts. The main results evidenced indicate the axes of the subject analyzed, the role of on-board nurses in the prehospital care during the aero-medical transport, evaluation of the patient's conditions in the gravid process, with adequate assistance based on protocols, besides the necessary skill and capacity for the removal and intercurrences in flight. Therefore aeromedical transport is an activity in development in Brazil, and its thematic must be deepened for its expansion of knowledge, corroborating to the rapid interventions in the process of removal of patient and state of emergency. Keywords: Airborne patient transport. Onboard nursing. Pregnancy process.

RECEBIDO EM: 25/1/2019

MODIFICAÇÕES REQUERIDAS EM: 1/7/2019

ACEITO EM: 5/7/2019

\footnotetext{
Enfermeira. Especialista em Assistência de Enfermagem ao Paciente em Estado Crítico - Faculdade Evangélica do Paraná (Fepar). Comunicação Organizacional - Universidade Estácio de Sá. Auditoria em Serviços de Saúde - Centro Universitário Internacional (Uninter). Mestre em Cirurgia - Pontifícia Universidade Católica do Paraná (PUC-PR). Docente titular no Departamento de saúde da Universidade Estácio de Sá - Curitiba. elia561@hotmail.com

${ }^{2}$ Enfermeiro. Especialista em Saúde do Idoso e Gerontologia - Faculdade Unyleya. Mestrando do Programa de Pós-Graduação em Saúde Coletiva Universidade Federal do Paraná (UFPR). wellingtonferreira42@gmail.com

${ }^{3}$ Geógrafo. Doutor em Geografia da Saúde pela Universidade Federal do Paraná (UFPR). Docente titular no Departamento de Enfermagem pelo Centro Universitário Campos de Andrade (Uniandrade). denecir.dutra@terra.com.br
} 


\section{INTRODUÇÃO}

A temática inerente ao transporte aeromédico consiste em mitigar à contemporaneidade o viés de uma alternativa imediata referente à condução de emergência ou urgência a paciente em iminência do estado crítico de vida, em uma gama variável de situações, constituindo-se esta a única alternativa de assistência em locais remotos de difícil acesso terrestre (FUIM, 2016; ALVARADO-SOCARRAS; IDROVO; BERMON, 2016).

Os principais aspectos desta alternativa de remoção imediata via espaço aéreo advêm do contexto histórico contemporâneo de batalhas e guerras, dada a necessidade de remoção rápida de feridos em áreas de batalhas bélicas em diferentes localizações do globo terrestre (COLDEBELLA; GRANDO; CAMBOIN, 2016). Mais precisamente, o contexto histórico aponta que em 1870 os esquadrões militares durante a Guerra Franco-Prussiana entre alemães e franceses, onde houvera registros de retiradas do campo de batalha de soldados machucados, por meio da utilização de balões de ar quente, como veículo aéreo agilizando o deslocamento de militares enfermos (CASTILLO; DE MEDEIROS, 2017; SLAVIERO et al., 2018).

Neste contexto, a Primeira Guerra Mundial proporcionou aspectos de assistência aos pacientes na modalidade aérea, por meio do transporte aeromédico, já com a participação dos profissionais de enfermagem. Na Segunda Guerra Mundial ocorreram locomoções nos aviões de carga, que continham leitos, acompanhadas por profissionais especializados da enfermagem e de saúde no atendimento, já naquela época, caracterizados como por "Flight Nurses" (DE LACERDA; DE MORAIS ARAÚJO; NETA, 2017; CONCEIÇÃO; SOARES, 2018).

A temática e demandas do transporte aeromédico, entretanto, proporcionaram um aumento em suas expectativas e desafios e em seu desenvolvimento principalmente em decorrência dos avanços tecnológicos da aviação e investimentos na habilitação de profissionais, nas primeiras décadas do século passado (COSTA et al., 2013; DOS SANTOS; GUEDES; AGUIAR, 2014; CARDOSO et al., 2017).

Nacionalmente iniciaram-se apenas na década de 60, por meio da Força Aérea Brasileira (FAB), pelos resgates emergenciais em helicópteros, principalmente em ocorrências de fatalidade aos aeronáuticos. Já para a população de civil a estatal brasileira Petrobras desenvolvia por meio de grupo de socorro de emergência junto ao corpo de bombeiros do Rio de Janeiro e patruIhamento aéreo da Polícia Militar, alternativas de remo- ção em situações de emergência, em suas plataformas bases no oceano (BONUZZl et al., 2016; ENCARNAÇÃO; MELO; LAGE, 2014; SCHWEITZER et al., 2017).

Somente em meados de 1990, contudo, insurgiu o uso do transporte aeromédico com os objetivos claros, em larga escala, dada a facilidade de remoção imediata a pacientes críticos e a existência de equipamentos avançados, proporcionando fácil acesso a serviços de saúde (FUIM, 2016; ALVARADO-SOCARRAS; IDROVO; BERMON, 2016).

Conforme as necessidades de remoção aérea a enfermos, aspectos inerentes ao processo são identificados como de grande valia para o desenvolvimento desta temática, tais como a existência de profissionais qualificados e especializados, equipe multiprofissional de bordo, enfermeiro e médico de bordo e o piloto (DE LIMA SOUZA et al., 2013; DIAS; FERREIRA; CARVALHO, 2017; LARA, 2018).

Deste modo, o profissional enfermeiro é evidenciado pela Associação de Emergência de Enfermagem (Emergency Nurses Association), e a Associação Nacional de Enfermeiros de Bordo (National Flight Nurses Association), nos Estados Unidos da América (EUA), respaldando e destacando a necessidade de habilidades e experiência em atuação com pacientes críticos emergenciais em remoção aérea (COLDEBELLA; GRANDO; CAMBOIN, 2016; CASTILLO; DE MEDEIROS, 2017; SLAVIERO et al., 2018).

No que se refere ao Brasil, a denominada enfermagem de bordo é caracterizada pela Lei n. 7.498/86, regulamentadora do Exercício Profissional da Enfermagem, a qual declara que são privativas do enfermeiro a organização e direção da assistência direta ao paciente crítico de maior complexidade técnica. Há ainda outra afirmativa de cunho legal ao profissional de enfermagem na Portaria GM 2.048, de 5 de novembro de 2002, que determina a capacitação específica dos profissionais de transporte aeromédico, referindo-se a aspectos inerentes à enfermagem. Já a Portaria n. 1.010, de 21 de maio de 2012, normatiza que a quantidade mínima de uma equipe aeromédica é de um médico e um enfermeiro (DE LACERDA; ARAÚJO; NETA, 2017; CONCEIÇÃO; SOARES, 2018).

Inerente a este contexto o Serviço de Atendimento Móvel de Urgência (Samu), instituído por meio das urgências e emergências, proporciona atendimento em residências, vias públicas, estruturando-se como transporte inter-hospitalar de pacientes, via terrestre, com equipes capacitadas, e inclui também aeronaves de transporte aeromédico necessário pela velocidade e versatilidade no atendimento ao paciente crítico (FERREIRA et al., 2017). A caracterização da 
aeronave, entretanto, é de espaço reduzido, em que o paciente é disposto na maca/prancha no sentido transversal durante a remoção. Os profissionais médico e enfermeiro posicionam-se ajoelhados no piso da aeronave (BONUZZI et al., 2016; ENCARNAÇÃO; MELO; LAGE, 2014; SCHWEITZER et al., 2017).

Deste modo, pacientes que se caracterizam como gestantes em processo gravídico evidenciado por meio de fenômeno fisiológico podem apresentar intercorrências durante a gestação e/ou no momento de parto. Embora as situações clínicas sejam variadas, dados apontam que cerca de $90 \%$ das gestações não oferecem complicações durante a evolução do processo gravídico, estas sendo definidas como gestações de baixo risco, entretanto há a possibilidade de ocorrerem as denominadas gestações de alto risco, concomitantemente à iminência de morte da mãe, do feto e/ ou do recém-nascido, pois são arriscadas as urgências e emergências obstétricas, que necessitam de resposta imediata, o que evidencia a importância da remoção aérea emergencial (FUIM, 2016; ALVARADO-SOCARRAS; IDROVO; BERMON, 2016).

No que tange às gestantes, determinadas manifestações clínicas, sinais e sintomas aparecem apenas no último trimestre da gestação, provocando condições ameaçadoras à vida da mãe, expondo as gestantes à necessidade de assistência especializada, a situações de urgências/emergências obstétricas, que exigem intervenções imediatas e, muitas vezes, de remoção aérea (DE LIMA SOUZA et al., 2013; DIAS; FERREIRA; CARVALHO, 2017; LARA, 2018).

Deste modo, intercorrências obstétricas comuns são hemorragia, principalmente a hemorragia pós-parto, que é a primeira causa de morte materna, acompanhada de infecção, e de doenças hipertensivas da gravidez, principalmente a eclâmpsia, considerada de elevado risco para urgências e emergências inerentes ao período gestacional (COLDEBELLA; GRANDO; CAMBOIN, 2016; CASTILLO; DE MEDEIROS, 2017; SLAVIERO et al., 2018).

Dessa forma, referente ao atendimento pré-hospitalar aéreo, o qual visa a diminuir óbitos, tempo de internação em hospitais, sequelas decorrentes de traumas em geral, e em situações que envolvem gestantes e recém-nascidos de alto risco, complicações obstétricas, através da prestação de um atendimento rápido e eficiente, faz-se temática relevante em seu aprofundamento (COSTA et al., 2013; DOS SANTOS; GUEDES; AGUIAR, 2014; CARDOSO et al., 2017).
Neste contexto, diante do exposto, a presente revisão buscou identificar as principais atribuições do profissional enfermeiro de bordo durante transporte aeromédico e sua dinâmica assistencial no processo gestacional em remoção.

\section{MÉTODOS}

Trata-se de uma revisão narrativa da literatura visando a reunir resultados da importância do profissional de enfermagem a bordo durante a remoção em transporte aeromédico relacionado a urgências em obstetrícia.

Nesse contexto, a revisão narrativa apresenta uma síntese pautada em diferentes tópicos, capazes de criar uma ampla compreensão sobre o conhecimento. Sendo assim, o primeiro passo para a construção do conhecimento científico surge por meio de novas teorias e da discussão de um assunto de pesquisa, lembrando que a revisão da literatura não é uma mera sumarização (BOTELHO; DE ALMEIDA CUNHA; MACEDO,, 2011).

Para ter acesso aos artigos explorados foram utilizados os descritores em ciências da saúde (DeCS): resgate aéreo; processo gravídico; medicina aeroespacial; enfermagem em emergência e cuidados de enfermagem.

O levantamento da base de dados da pesquisa foi realizado em bibliotecas públicas e acervos de dados digitais como: Literatura Latino-Americana e do Caribe em Ciências da Saúde (Lilacs), Centro Latino-Americano e do Caribe de Informação em Ciências da Saúde (Bireme), Scientific Eletronic Library Online (SciELO), Biblioteca Virtual em Saúde (BVS), Scholar (Google Acadêmico) e Revistas de Enfermagem.

Foram incluídos na pesquisa artigos originais com disponibilidade do texto completo em suporte eletrônico, sendo estabelecida a utilização de textos publicados desde 2013 ao primeiro semestre de 2018 dentro do foco de interesse, disponibilizados como Brasil no critério País/Região de assunto. O recorte temporal justifica-se pela relevância dos últimos apontamentos sobre a temática.

Os critérios de exclusão foram: artigos de reflexão, publicações cujo tema principal não correspondia à pesquisa, artigos internacionais, artigos duplicados em termos de conteúdos nas diferentes bases de dados. 
Assim, o material foi composto de 16 artigos que foram submetidos à técnica de avaliação e análise de conteúdo constituído por três etapas: pré-análise, exploração do material e interpretação dos resultados (BOTELHO; DE ALMEIDA CUNHA; MACEDO, 2011).

A primeira etapa possibilitou uma visão geral do conteúdo dos artigos, por meio da leitura dos resumos e fichamento. Os textos na íntegra, após uma primeira leitura, foram organizados com o auxílio de um formulário composto das variáveis: ano/autor, objetivos e resultados encontrados, descritos em discussão no decorrer do estudo.

A etapa de exploração do material foi desenvolvida a partir da releitura dos textos, culminando na construção de categorias temáticas de análise. Posteriormente, na etapa de interpretação dos resultados, foram observadas as colocações existentes sob a ótica de diferentes autores.

\section{Figura 1 - Fluxograma do processo de seleção dos estudos}
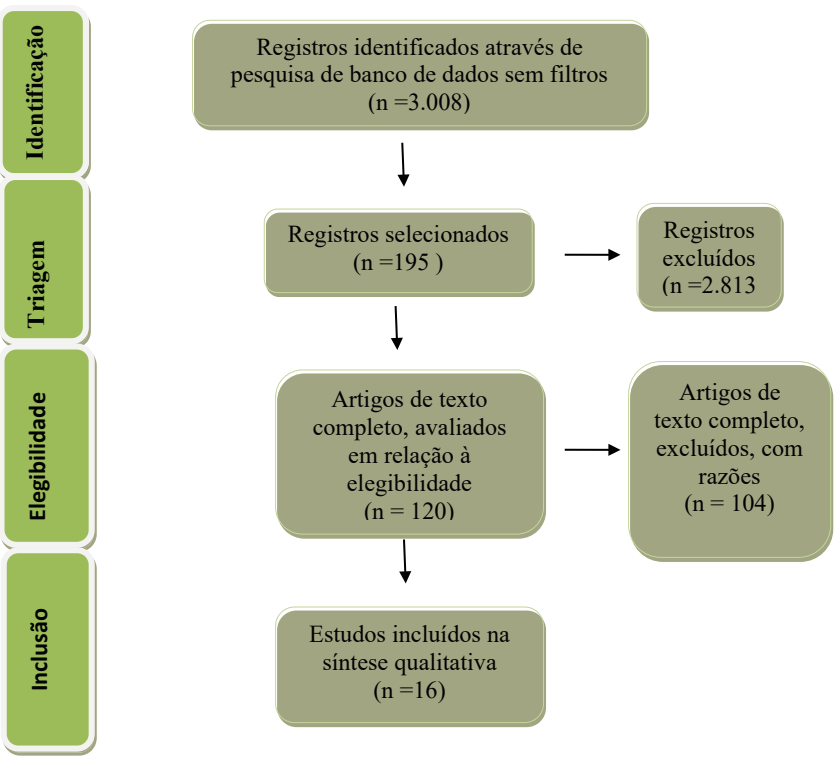

Fonte: Os autores.

\section{RESULTADOS E DISCUSSÕES}

Os achados obtidos por meio da pesquisa apontam uma produção atual escassa nas dimensões propostas desta temática. Em linhas gerais destacaram-se trabalhos científicos voltados a eixos fundamentais: resgate aéreo, processo gravídico, enfermagem aeroespacial de bordo, enfermagem em emergência, cuidados de enfermagem, protocolos aéreos.

Referente ao transporte aeromédico, caracteriza-se como uma modalidade de deslocamento de paciente empregado, sobretudo, para assistência de enfermos em estado crítico em urgência e emergência. A temática do enfermeiro de bordo ainda é recente em nosso país, existindo poucos cursos específicos na área, ou seja, formação voltada para a emergência em aeronaves. $O$ paciente removido por via aérea deve estar preparado para que lhe sejam empregados todos os recursos tecnológicos disponíveis na aeronave, mantendo-o estabilizado durante todo o voo. Para o profissional é necessário um vasto conhecimento sobre fisiologia de voo. Por isso a importância de uma avaliação constante, seguindo protocolos mundialmente aceitos, como o Pré-Hospitalar Trauma Life Support (PHTLS) (COLDEBELLA; GRANDO; CAMBOIN, 2016; CASTILLO; DE MEDEIROS, 2017; SLAVIERO et al., 2018).

Em relação aos aspectos inerentes às situações avaliadas como urgentes, um fator preponderante e de extrema relevância é o tempo entre o acontecimento da ocorrência e o atendimento, por meio da modalidade de serviço empenhado no socorro. Deste modo, os serviços pré-hospitalares, tanto o móvel terrestre quanto o aéreo de urgência, caracterizam-se pelo acesso rápido e eficaz ao serviço de saúde, contribuindo para um bom prognóstico de saúde da população usuária (FUIM, 2016; ALVARADO-SOCARRAS; IDROVO; BERMON, 2016).

Neste contexto, considera-se as especificidades e a importância da área obstétrica no que tange à assistência no transporte aeromédico. Para contextualizar a temática dados da Organização Mundial da Saúde (OMS) apontam que em 2010 ocorreram aproximadamente 287.000 óbitos maternos em âmbito global. Já no Brasil, segundo o Departamento de Informática do SUS (Datasus), via Sistema de Informações sobre Mortalidade (SIM), constatou-se 1.567 óbitos maternos em 2013 em decorrência de intercorrências durante o processo do parto (COSTA et al., 2013; DOS SANTOS; GUEDES; AGUIAR, 2014; CARDOSO et al., 2017).

Reafirmando a problemática, dados do Ministério da Saúde (MS) referem-se a mortes maternas decorrentes de episódios malsucedidos, como carência de acolhimento à gestante e/ou puérpera, ausência de suporte familiar ou social e dos serviços de saúde (BONUZZI et al., 2016; ENCARNAÇÃO; MELO; LAGE, 2014; SCHWEITZER et al., 2017).

Nesse contexto, a correta remoção das pacientes durante intercorrências no processo gravídico deve ser realizada conforme queixas e sintomas a um acesso adequado, visando a não agravar o panorama de socorro das urgências e emergências obstétricas. Assim, as patologias ou intercorrências que agravam a saúde de gestantes e puérperas são evidenciadas 
por meio de infecções, hipertensão arterial prévia ou atual, hemorragias, cardiopatias, asma aguda grave e distúrbios tromboembólicos, além de violências urbanas e acidentes automobilísticos, entre outras (DE LIMA SOUZA et al., 2013; DIAS; FERREIRA; CARVALHO, 2017; LARA, 2018).

Nesse contexto, o atendimento de urgência gestacional exige rapidez, pronto atendimento, uma adequada avaliação do quadro e as alternativas de suporte disponíveis no serviço de emergência, as quais são essenciais para o desfecho adequado da intercorrência (COLDEBELLA; GRANDO; CAMBOIN, 2016; CASTILLO; DE MEDEIROS, 2017; SLAVIERO et al., 2018).

Assim sendo, compreender as características das ocorrências obstétricas atendidas por serviço de urgência torna-se fundamental, influenciando nas condutas profissionais e assistências de prevenção a agravos, inerente ao processo de remoção no transporte aereomédico (FUIM, 2016; ALVARADO-SOCARRAS; IDROVO; BERMON, 2016).

O fenômeno mais frequente identificado para a utilização do transporte aeromédico é o parto, que gera grande número de atendimentos de urgência obstétrica, os quais estão relacionados diretamente ao risco de óbito materno-fetal, além de pequena proporção de gestantes vítimas de acidentes de trânsito e agressão física e remoção do neonato a emergências em deslocamento a instituições hospitalares mais bem equipadas, oriundos de diferentes regiões do país com menor infraestrutura médica hospitalar (BONUZZI et al., 2016; ENCARNAÇÃO; MELO; LAGE, 2014; SCHWEITZER et al., 2017).

Desse modo, juntamente com o transporte aeromédico, o atendimento apropriado para grávidas é específico, com o melhor manejo no atendimento pré-hospitalar ao binômio mãe/filho que deve prevenir agravos severos que culminem na morte materna e igualmente na redução de chance de intercorrências e óbitos neonatais (DE LACERDA; De MORAIS ARAÚJO; NETA, 2017; CONCEIÇÃO; SOARES, 2018).

Logo, no atendimento pré-hospitalar, o uso de helicópteros aeromédicos equipados com suporte de vida, torna provável a sobrevivência de gestantes em estado crítico, em remoções de urgência ou atendimento inicial da ocorrência e estando a sua presença e utilização em lugares cada vez mais remotos (COLDEBELLA; GRANDO; CAMBOIN, 2016; CASTILLO; DE MEDEIROS, 2017; SLAVIERO et al., 2018).

O processo assistencial do profissional de enfermagem de bordo durante o trajeto da remoção, por via aérea, é caracterizado por sua atribuição principal, ou seja, ajustar e/ou diminuir as implicações da altitude no organismo, efeitos das forças gravitacionais durante funcionamento da aeronave, monitorando possíveis intercorrências, visando a preservar a vida, afastando riscos e/ou operacionando nestes (COSTA et al., 2013; DOS SANTOS; GUEDES; AGUIAR, 2014; CARDOSO et al., 2017).

Desse modo, existem para o melhor manejo na remoção aérea protocolos que direcionam a assistência de enfermagem durante e após o voo, elaborados para enfermeiros de bordo atuantes no atendimento a pacientes obstétricas, os quais orientam a segurança de voo de helicópteros e a avaliação durante o transporte aeromédico (DE LACERDA; DE MORAIS ARAÚJO; NETA, 2017; CONCEIÇÃO; SOARES, 2018).

Em linhas gerais as assistências estão organizadas por meio da sequência linear de procedimentos, necessários para priorizar fatores durante o atendimento em relação ao aspectos do processo de fisiologia de voo destas pacientes destacando-se que grande parte desses procedimentos podem ser realizados simultaneamente (BONUZZl et al., 2016; ENCARNAÇÃO; MELO; LAGE, 2014; SCHWEITZER et al., 2017).

Em suma, para seguir o protocolo de remoção a uma população específica durante o voo, deve-se atentar ao atendimento das vias aéreas e controle da coluna cervical, caso o trauma ou emergência ocorra com um gestante. Assim, é necessário deixar a prancha rígida ligeiramente inclinada para a esquerda, a cabeça, o pescoço, o tronco e a pelve devem ser imobilizados em posição alinhada e neutra, para impedir qualquer movimento de coluna. Essa posição facilita a avaliação constante. Algumas gestantes tendem a apresentar hipotensão elevada proeminente; esta condição pode ocorrer no 30 trimestre e é motivada pela constrição do útero sobre a veia cava inferior, diminuindo expressivamente o regresso venoso para o coração e, como há menor enchimento do vaso, o débito cardíaco e a pressão arterial diminuem (DE LIMA SOUZA et al., 2013; DIAS; FERREIRA; CARVALHO, 2017; LARA, 2018).

Outro ponto dos protocolos do transporte aeromédico a que se deve atentar diz respeito ao sistema respiratório. Deve-se realizar a monitorização da oximetria de pulso e administração de oxigênio para prevenir a hipóxia de altitude em pacientes entubados, traqueostomizados, em ventilação mecânica ou com reanimador manual quando necessário. Essa ação se justifica pelo fato da identificação na atmosfera de níveis que impõem graus progressivos de hipóxia ao organismo com consequentes alterações fisiológicas. Autores apontam que de 0 a 6.000 pés quase nenhu- 
ma alteração fisiológica é observada. A saturação de hemoglobina, que é de $98 \%$, cai para $87 \%$ a 10.000 pés, e a $60 \%$ a 22.000 pés, portanto, os parâmetros ventilatórios devem ser frequentemente reavaliados durante o voo (FUIM, 2016; ALVARADO-SOCARRAS; IDROVO; BERMON, 2016).

Parâmetro importante a ser observado durante o transporte aeromédico é a circulação do paciente com o monitor de multiparâmetros, equipamento que permite o acompanhamento hemodinâmico, simultâneo, de parâmetros vitais como: pressão arterial, frequência cardíaca, saturação de oxigênio. Sem esta tecnologia não seria possível a mensuração devido ao alto ruído presente no interior do helicóptero, que chega a mais de 110 decibéis. Administrar medicamentos por meio de soro em pressurizador e medicamentos endovenosos em bomba de seringa, pois a altitude e suas variações durante o voo dificultam e interferem nas tentativas de infusões endovenosas realizadas por gravidade, tudo isso para dar suporte à gestante ou ao neonato, conforme a ocorrência. Há ainda incubadora para os neonatos dentro da aeronave (COLDEBELLA; GRANDO; CAMBOIN, 2016; CASTILLO; DE MEDEIROS, 2017; SLAVIERO et al., 2018).

Alguns autores relatam que há 11 principais fatores de estresse presentes durante um transporte aeroespacial: hipóxia, disbarismos (alterações na pressão do ambiente), umidade do ar, temperatura, vibrações, ruído, forças acelerativas e gravitacionais, luminosidade, sobrecargas musculoesqueléticas, fadiga de voo e fusos horários (DE LACERDA; DE MORAIS ARAÚJO; NETA, 2017; CONCEIÇÃO; SOARES, 2018).

$O$ fato de manter a gestante corretamente posicionada na prancha rígida diminui o desconforto, aumenta a segurança da paciente durante o voo e minimiza os efeitos das forças gravitacionais. Proteger os ouvidos com abafador de orelha ( ruídos intensos provocam desconforto), manter a paciente aquecida com manta térmica e cobertor, pois a cada 1.000 pés a temperatura cai $2^{\circ} \mathrm{C}$ (COSTA, et al. 2013; DOS SANTOS; GUEDES; AGUIAR, 2014; CARDOSO et al., 2017).

Neste contexto, para os profissionais enfermeiros de bordo no atendimento pré-hospitalar aéreo, é necessário aceitar a responsabilidade de cuidar do paciente, com estudo e dedicação no atendimento, pois na remoção durante o transporte aeromédico não há muito tempo e por isso suas funções específicas devem ser realizadas de maneira eficiente e adequada para o suporte de vida das gestantes nas emergências. Desta forma, intensifica-se a importância de protoco- los de cuidados que orientem o profissional durante a assistência (FUIM, 2016; ALVARADO-SOCARRAS; IDROVO; BERMON, 2016).

As orientações de segurança durante o voo de helicóptero ou aeronaves, em suma, consistem em fixar o soro e/ou outros equipamentos junto ao paciente e sobre a maca rígida ao levá-lo para a aeronave, e não deixar o lençol ou manta térmica soltos, monitorar o paciente e intervir com manobras necessárias. Deve-se atentar a conversas entre a equipe de voo, que precisam ser objetivas para não haver interferência de comunicação. É necessário obter informações concretas sobre o voo, principalmente o tempo de deslocamento, com o piloto, para planejar a assistência prestada; o profissional enfermeiro deve ser informado acerca das mudanças de rota de voo/ tempo para esquematizar o volume de medicação e o consumo de oxigênio, de acordo com o estado clínico do paciente e/ou ocorrência (DE LIMA SOUZA et al., 2013; DIAS; FERREIRA; CARVALHO, 2017; LARA, 2018).

Em relação à gestante, neonato ou demais pacientes traumatizados em remoção aérea, após o voo deve-se passar o plantão para o enfermeiro em terra sobre os cuidados de enfermagem realizados, os sinais vitais iniciais, glasgow, os procedimentos efetuados, as intercorrências, como foi o transporte e informar presença ou não de familiar. Preencher toda a ficha de atendimento e solicitar a assinatura do médico responsável pelo paciente no hospital (COLDEBELLA; GRANDO; CAMBOIN, 2016; CASTILLO; DE MEDEIROS, 2017; SLAVIERO et al., 2018).

Isso posto, seguir protocolos de cuidados preconizados durante o voo aeromédico, priorizar ou organizar as ações de enfermagem a gestantes ou neonatos no ambiente aeroespacial, torna-se um importante instrumento na tomada de decisão, sistematizando a assistência prestada, possibilitando uma avaliação, evitando a repetição de erros, evidenciando o papel do enfermeiro de bordo no cuidado do paciente durante a remoção aérea.

\section{CONSIDERAÇÕES FINAIS}

A presente revisão apresentou como principal achado a reflexão e compreensão dos aspectos inerentes à remoção aeromédica, em especial ao transporte de pacientes em processo gravídico e neonatos. Evidenciou-se uma solução dinâmica de benefícios à assistência de pacientes graves em situação de emergência. 
Nesse contexto, como principal limitação da temática foram as poucas pesquisas voltadas para a atuação do enfermeiro no transporte aeromédico, por ser um tema ainda recente quanto aos aspectos específicos propostos como objetivos e/ou pelo alto custo. Determinadas publicações estão desatualizadas, tendo sido publicadas há mais de uma década.

Como contribuição para a prática profissional esta revisão ressalta a necessidade de preencher essa lacuna, proporcionando uma atenção para tal temática, propondo efetividade da assistência de enfermagem e capacitação profissional. A assistência aeroespacial a gestantes deve embasar-se principalmente nas alterações fisiológicas decorrentes do voo e protocolos supracitados.

Assim sendo, o emprego de protocolos torna-se instrumento na tomada de decisão do enfermeiro de bordo, priorizando e organizando ações práticas e efetivas de enfermagem. Diante disto, espera-se que informações lançadas com a presente pesquisa, ainda que embrionárias, instiguem enfermeiros de bordo e/ ou instituições acadêmicas e profissionais à reflexão de sua prática e sirvam de subsídios para novos estudos que abarquem a temática.

\section{REFERÊNCIAS}

ALVARADO-SOCARRAS, Jorge Luis; IDROVO, Alvaro Javier; BERMON, Anderson. Sobrevida hospitalar após a alta de neonatos doentes transportados por ambulância terrestre ou aérea para um centro terciário. Jornal de Pediatria, v. 92, n. 3, 2016.

BONUZZI, Karen Leme et al. Atuação do enfermeiro no atendimento pré hospitalar aéreo a pacientes politraumatizados-Revisão de literatura. Revista de Divulgação Científica Sena Aires, v. 5, n. 2, p. 171-177, 2016.

BOTELHO, Louise Lira Roedel; DE ALMEIDA CUNHA, Cristiano Castro; MACEDO, Marcelo. O método da revisão integrativa nos estudos organizacionais. Gestão e Sociedade, v. 5, n. 11, p. 121-136, 2011.

CARDOSO, Ricardo Galesso et al. Análise da aplicação dos conceitos de Corporate Resource Management (CRM) nas missões de resgate aeromédico no grupamento de radiopatrulha Aérea João Negrão (GRPAE). Revista Conexão Sipaer, v. 8, n. 2, p. 13-20, 2017.

CASTILLO, Vanessa Dina Palomino; DE MEDEIROS, Clarissa Mari. Emergências médicas em voos comerciais: uma revisão de literatura. Saúde, Ética \& Justiça, v. 21, n. 1, p. 18-27, 2017.
COLDEBELLA, Vanessa; GRANDO, Liandra Kasparowiz; CAMBOIN, Franciele Foschiera. Atendimento neonatal: serviço aeromédico Paraná Urgência/Samu base Cascavel. Espaço para a Saúde - Revista de Saúde Pública do Paraná, v. 17, n. 2, p. 143-151, 2016.

CONCEIÇÃO, Aldo Nery; SOARES, Marcelo Falcão. Serviço aeropolicial na pmto: proposta de reativação. Aturá-Revista Pan-Amazônica de Comunicação, v. 2, n. 3, p. 326-347, 2018.

COSTA, Nayara Mendes et al. a ótica empreendedora do enfermeiro: capacitação e atuação de profissionais no transporte aeromédico. NBC-Periódico Científico do Núcleo de Biociências, v. 3, n. 5, p. 39-49, 2013. Disponível em: http:// dx.doi.org/10.15601/2238-1945/pcnb3n5p39-49.

DE LACERDA, Leticia Soares; DE MORAIS ARAÚJO, Eronice Ribeiro; NETA, Francina Lopes Amorim. Transporte aeromédico no estado do Piauí: perfil das ocorrências. Revista Prevenção de Infeç̧ão e Saúde, v. 3, n. 2, p. 20-26, 2017.

DE LIMA SOUZA, Wbiratan et al. Evolução sócio-histórica da saúde aeroespacial com enfoque na enfermagem: revisão integrativa. Caderno de Graduação-Ciências Biológicas e da Saúde-Unit-Alagoas, v. 1, n. 2, p. 165-176, 2013.

DIAS, Carla Pena; FERREIRA, Flávio Lopes; CARVALHO, Vânia Paula de. A importância do trabalho em equipe no transporte aéreo de pacientes. Rev. Enferm. UFPE on-line, v. 11, n. 6, p. 2.408-2.414, 2017.

DOS SANTOS, Heloisa Griese Luciano; GUEDES, Carolina Cristina Pereira; AGUIAR, Beatriz Gerbassi Costa. A segurança do paciente no transporte aeromédico: uma reflexão para a atuação do enfermeiro. Revista Acreditação: Acred, v. 4, n. 7, p. 21-34, 2014.

ENCARNAÇÃO, Paula Soares; MELO, Maria Manuela; LAGE, Maria Isabel. A pessoa em situação crítica helitransportada: história do passado recente e panorama atual. Revista de Enfermagem Referência, n. 2, p. 171-183, 2014.

FERREIRA, Wellington Fernando da Silva et al. Urgência e emergência nas dimensões da pessoa idosa: Sistema Manchester. Revista Saúde e Desenvolvimento, v. 11, n. 6, p. 283-293, 2017.

FUIM, Edson Fernando. Perfil dos pacientes atendidos em um serviço de transporte aeromédico privado. Sínteses: Revista Eletrônica do Simtec, n. 6, p. 222, 2016.

LARA, Marcos Oliveira. O emprego do helicóptero no atendimento pré-hospitalar. O Alferes, v. 21, n. 59, 2018.

SCHWEITZER, Gabriela et al. Intervenções de emergência realizadas nas vítimas de trauma de um serviço aeromédico. Revista Brasileira de Enfermagem, v. 70, n. 1, p. 54-60, 2017.

SLAVIERO, Raffael Sehn et al. Perfil epidemiológico dos pacientes atendidos no período de 2014 a 2016 pelo serviço de transporte aeromédico interhospitalar vinculado ao con- 